\title{
Prevalence of Goiter and Associated Factors Among Primary School Children Aged 6-12 Years Old in Goba Town, South East, Ethiopia
}

\author{
Habtamu Demelash Enyew ${ }^{1,}$, Ketema Gashaw Zemedkun², Addisu Melese Dagnaw ${ }^{3}$ \\ ${ }^{1}$ Department of Public Health, College of Medicine and Health Sciences, Madawalabu University, Bale Goba, Ethiopia \\ ${ }^{2}$ Department of Nursing, College of Medicine and Health Sciences, Madawalabu University, Bale Goba, Ethiopia \\ ${ }^{3}$ Department of Medicine, College of Medicine and Health Science, Madawalabu University, Bale Goba, Ethiopia \\ Email address: \\ hab2396@yahoo.com (H. D. Enyew),ket1328@yahoo.com (K. G. Zemedkun), addisum22@gmail.com (A. M. Dagnaw)
}

\section{To cite this article:}

Habtamu Demelash Enyew, Ketema Gashaw Zemedkun, Addisu Melese Dagnaw. Prevalence of Goiter and Associated Factors Among Primary School Children Aged 6-12 Years Old in Goba Town, South East, Ethiopia. International Journal of Nutrition and Food Sciences. Vol. 4, No. 3, 2015, pp. 381-387. doi: 10.11648/j.ijnfs.20150403.26

\begin{abstract}
Background: There are many reasons for children to underperform at school such as nutritional status, sociocultural environment and other environmental factors. Iodine deficiency disorder is the major cause of preventable brain damage in children resulting in academic underperformance. Although few studies have been conducted on goiter among primary school children in Ethiopia, the mountainous area like Goba town which experiences poor soil conservation over a long period of time and the leaching away of the iodine-rich soil, needs special concern. Objective: This study assessed the prevalence of goiter and its associated factors among school children in Goba town, southeastern Ethiopia. Methods: School based cross sectional study design was employed on study participants of 397 children in age groups of 6-12 years from four primary schools in Goba town with a 98.2\% response rate. The study was conducted from April to May, 2012. A systematic sampling technique was applied to select children; Pre-tested structured questionnaire was used to collect data on sociodemographic variables of children and their parents. Selected students were examined for the presence of goiter and classified based on WHO recommendation. Salt test for iodine content was done using field fast salt test kit. Multiple logistic regression analysis was done to see the effect of independent variables on the outcome variable (presence of goiter). Results: Out of 397 childern 209(52.6\%) were females. The total goiter prevalence was 50.6\%\{95\% CI $=(45.7,55.6)\}$ with grade 1 goiter having $33.5 \%\{95 \% \mathrm{CI}=(28.9,38.4)\}$ and grade 2 goiter having $17.1 \%\{95 \% \mathrm{CI}=(13.6,21.3)\}$. The goiter prevalence was found higher in females $112(53.6 \%)\{95 \% \mathrm{CI}=(46.6,60.5)\}$ than in males $89(47.3 \%)\{95 \% \mathrm{CI}=(40.0,54.7)\}$ though statistically insignificant in the studied school age children. The age of study subjects (AOR=1.86; 95\% CI=1.10, 3.15) had significant association with goiter. With respect to salt consumption at home, for the study participants $110(27.7 \%)$ of children were given salt which had no iodine $(0 \mathrm{ppm})$ and $169(42.6 \%)$ of them were given salt which contained medium level of salt $(<15 \mathrm{ppm})$ and the remaining $118(29.7 \%)$ were given sufficiently iodized salt. Conclusion: Prevalence of total goiter among school children is very high and it was higher in females than males. The salt test results showed that most households do not use sufficiently iodised salt.
\end{abstract}

Keywords: Iodine, Goiter, Children, Iodized Salt

\section{Background}

Enlargement of the thyroid gland (whose production of hormones requires iodine) is the best-known sign of iodine deficiency, called goiter. The worst effect is cretinism severe mental retardation [1]. Iodine deficiency is one of the most common preventable causes of mental retardation in the world today [2]. It is a global problem, the endemic area being located in the mountainous region of Europe, Asia, south and Central America and eastern Africa [3]. Recent studies in school children in Egypt, Swaziland and South Africa showed that the overall prevalence rate of iodine deficiency disorder (IDD) was between $35 \%$ and $70 \%$ indicating a severe public health problem in each of the areas studied [4]. Ethiopia is a mountainous country, and poor soil conservation over a long period may have contributed to the 
leaching away of the iodine-rich soil layer and the exposing of the iodine-poor layer beneath. The highest rates of palpable and visible goiter was found in the south nation nationalities and people (SNNP) regional state (56.2\%), followed by $42.0 \%$ in Oromia, $40.5 \%$ in Benshangul Gumuz, $29.1 \%$ in Amhara, and $21.9 \%$ in Tigray. The prevalence goiter rate was $40 \%$ in school age children and $36 \%$ in mothers [5]. Studies showed that two of the iodine deficiency disorder indicators (goiter and urinary iodine excretion) were significantly associated with the level of intelligence of the school-children, which reflected the quality of life of the people [6]. The principal cause of iodine deficiency is inadequate iodine in foods. Since iodine cannot be stored for long periods by the body, tiny amounts are needed regularly (100-150 micrograms per day per person).The Ethiopian demography and health survey (EDHS 2011) results showed that only 15 percent of children 6-59 months live in households using adequately iodized salt [7].

According to the World Health Organization (WHO), a total goitre rate of $5 \%$ or more in primary-school children (612 years) should signal the presence of a public health problem [4].The aim of this study was to assess the prevalence of goiter and its determinant factors among school children in Goba town southeastern Ethiopia.

\section{Methods}

The school based cross sectional study was conducted in Goba town from April to May 2012. The town has one preparatory school, one high school, nine elementary schools and ten Kindergarten schools (Goba education office). Children in the age range 6-12 years were included in the study and those who were ill excluded from study. As per recommendation of WHO/UNICEF/ICCIDD, the school children in the age group 6-12 years were selected, because of their high vulnerability to goiter, easy accessibility and because of their representativeness of their age group in the community [4]. Sample size was calculated using single population proportion formula by assuming the prevalence of goiter in children aged 6-12 years 39.9\% [3], with 95\% confidence interval, 5\% absolute precision, $10 \%$ nonresponse rate, the total sample size was 404 . The required sample size was taken according to the size of students aged 6-12 years in each school.

The number of schools in the town was obtained from Goba town education office. The consent was obtained from the town school authority and perspective schools. Four schools were selected randomly from nine primary schools in the town.The number and list of students was obtained from each selected school. Proportion allocation was done to determine the number of students that has to be included in the study from each school based on the number of students. The same procedure was followed to determine the number of students in each class room of the school. Finally each school child was selected using systematic sampling technique from list of students in each class room. A student absent on the date of data collection was substituted by the next student from the same class room.

\subsection{Data Collection Tools and Techniques}

Questionnaire based interview was undertaken by a trained interviewer with each child to obtain information on socio economic status. Data was collected using a pre-tested structured questionnaire prepared by reviewing prior study and other materials on the topic. The questionnaire was translated into Amharic language and back to English to ensure its consistency. The interview was conducted by four Bachelor of Science degree (BSc) nurses. A one day training was given to data collectors by the investigators. Data collectors approached children by first introducing themselves, and then explaining the purpose of their visit by reading the information sheet, after which they collected information. Those children who were unable to give a response to questions concerning family educational status and income, were asked to return with their parents the following day for interview.

Parents were interviewed after they were asked their permission orally to give the information. Continuous supervision of the data collection process was carried out.

Selected students of the recommended age group were clinically examined for the enlargement of thyroid (goiter) by two trained health officers to minimize inter-individual variability using palpation method as per recommendations. The health professionals who did the goiter examinations were recruited from Goba hospital and, were routinely exposed such types of activities both on the job and in various trainings given by non-governmental organizations.Goiter grading was done as per recommendation of WHO/UNICEF [Grade 0: no goiter; Grade 1: thyroid palpable but not visible; Grade 2: thyroid visible with neck in normal position]. In this particular study, the presence of goiter was considered absent if no palpable or visible goiter (Grade 0), whereas goiter is present when the child has Grade 1 or grade 2 goiter or both. The other quantifiable indicators like UIE method and TSH were not considered in the study because they are costly and inaccessible in our settings.

Children were asked to bring salt one day before the interview. Those who failed to bring salt for the first day were interviewed and reinstructed to bring the salt for the next day.The iodine content of salt was checked by iodized salt field test kit. The procedure was filling a small cup with salt, then spreading the salt on a flat surface. Two drops of the test solution were then added to the surface of the salt by piercing the white ampoule with a pin and gently squeezing the ampoule. The color acquired by the salt was then compared with the color chart within 1 minute in order to determine the iodine content. The results were expressed in parts per million (ppm). iodine content of salt[ sufficient $>$ $15 \mathrm{ppm}$, medium $<15 \mathrm{ppm}$ and no iodine $0 \mathrm{ppm}]$.

\subsection{Data Quality Management}

Data quality assurance was done during questionnaire 
designing, data collection and data entry. Questionnaire was objective, logically sequenced and free of scientific terms, non-leading and pretested. The data collectors were provided with intensive training on the objective of the study and on areas like thyroid examination to minimize inter-individual variability. The collected data was checked by the Principal investigator on a daily basis for any incompleteness and/or inconsistency. If any incompleteness and/or inconsistency appeared, correction was made by going back to the child or by taking the appropriate measure.

\subsection{Data Processing and Analysis}

First, the completeness of the questionnaires was checked. Then data from the completed questionnaires were entered into Epi-Info version 3.5.1 and exported to SPSS version 16 for analysis. The data were summarized and presented in the form of text, tables and figures. Binary and multiple logistic regression analyisis were performed to identify associated factors with goiter. The presence of association was determined by odds ratio with $95 \%$ confidence interval. Pvalues less than 0.05 were considerded as significant.

\subsection{Ethical Considerations}

Ethical clearance was obtained from the research ethics and reviewing committee of Madawalabu University, college of health science. Permission was obtained from Goba town education offices and the school directors of each school. Because of difficulty of meeting the student's family, the investigators discussed the ethical issues with the school officials. The study had no harm to the students, their families nor the school environment. Therefore, the involvement of parents was not considered as a prerequisite in this study. The freedom of children to participate or not participate in the study was explained and respected. Those children who were identified having goiter during data collection were advised to visit the nearby health facility to obtain possible support and treatment.

\section{Results}

From the total sample size of 404 children, 397 participated in this study giving $98.2 \%$ response rate. Two hundred nine $(52.6 \%)$ children were females. A greater proportion $315(79.3 \%)$ of the study subjects were in the age group 9-12 years. Mean age of the study subjects was 10 years. ( $\mathrm{SD}=1.62)$. Majority, $279(70.3 \%)$ of children were Orthodox Christian by religion. More than half, 230 (57.9\%) of the study subjects were from Oromo ethnic group, followed by Amhara 134 (33.8\%). Most, 278(70.0\%) of the children were born in Goba town, while 119 (30.0\%) were born elsewhere.

Regarding the occupational status of parents, the majority, $164(44.3 \%)$ of children's fathers were government employees followed by private sector workers, 73(19.3\%). However, most of the mothers of the study subjects were housewives, 198(50.1\%) followed by merchants, $88(22.3 \%)$ (Figure1).

With respect to the educational status of the parents, the majority, $161(43.5 \%)$ of the children's fathers attended school between 9-12 grade followed by 103 (27.8\%) fathers who completed above 12 grade. Out of 395 children's mothers, 168 (42.5\%) completed grade nine up to twelve, and $95(24.1 \%)$ had completed grade one up to eight (Figure 2 ).

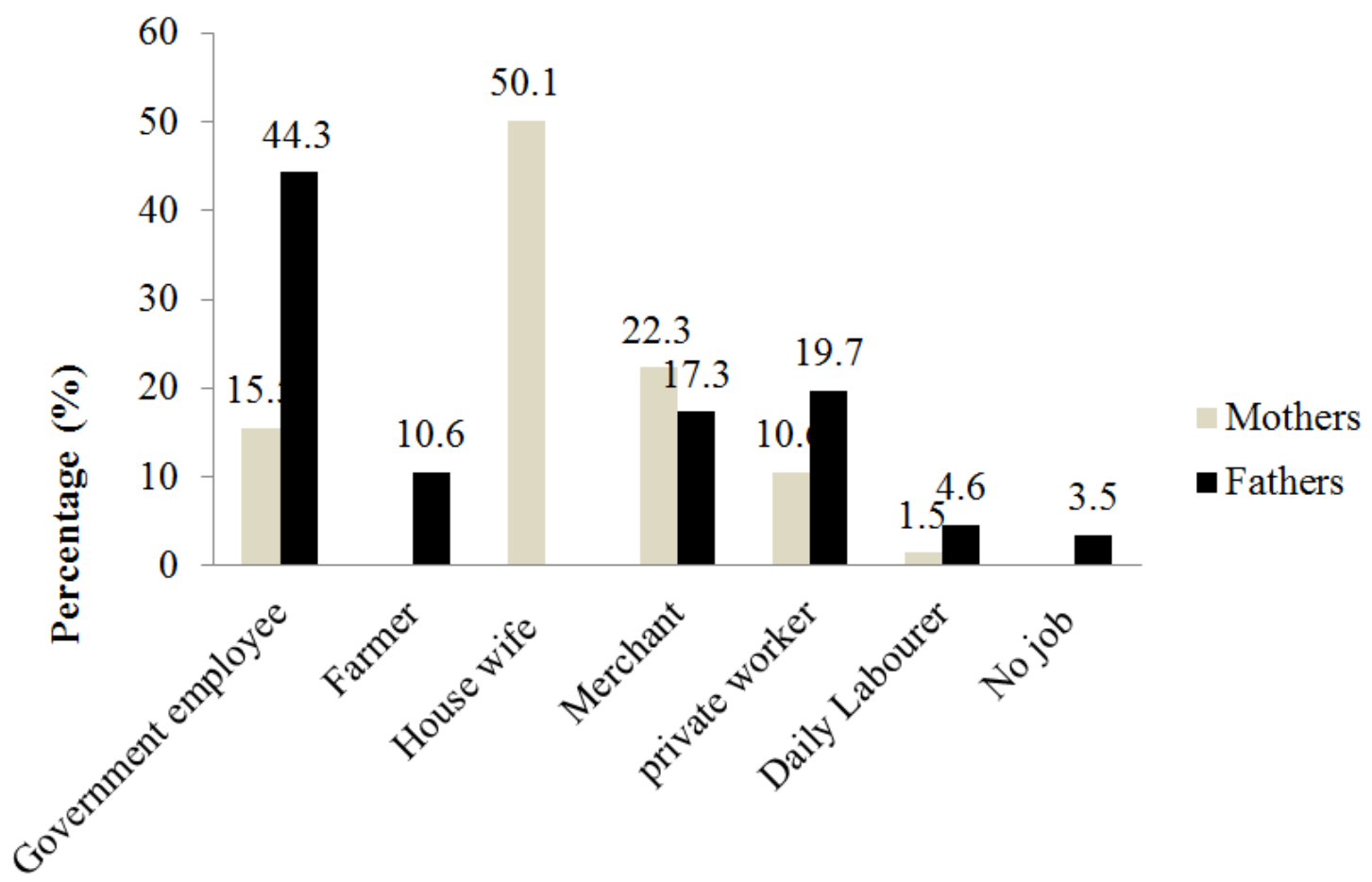

Fig. 1. The distribution of children by their parents' educational status in Goba town, South eastern Ethiopia, May, 2012. 


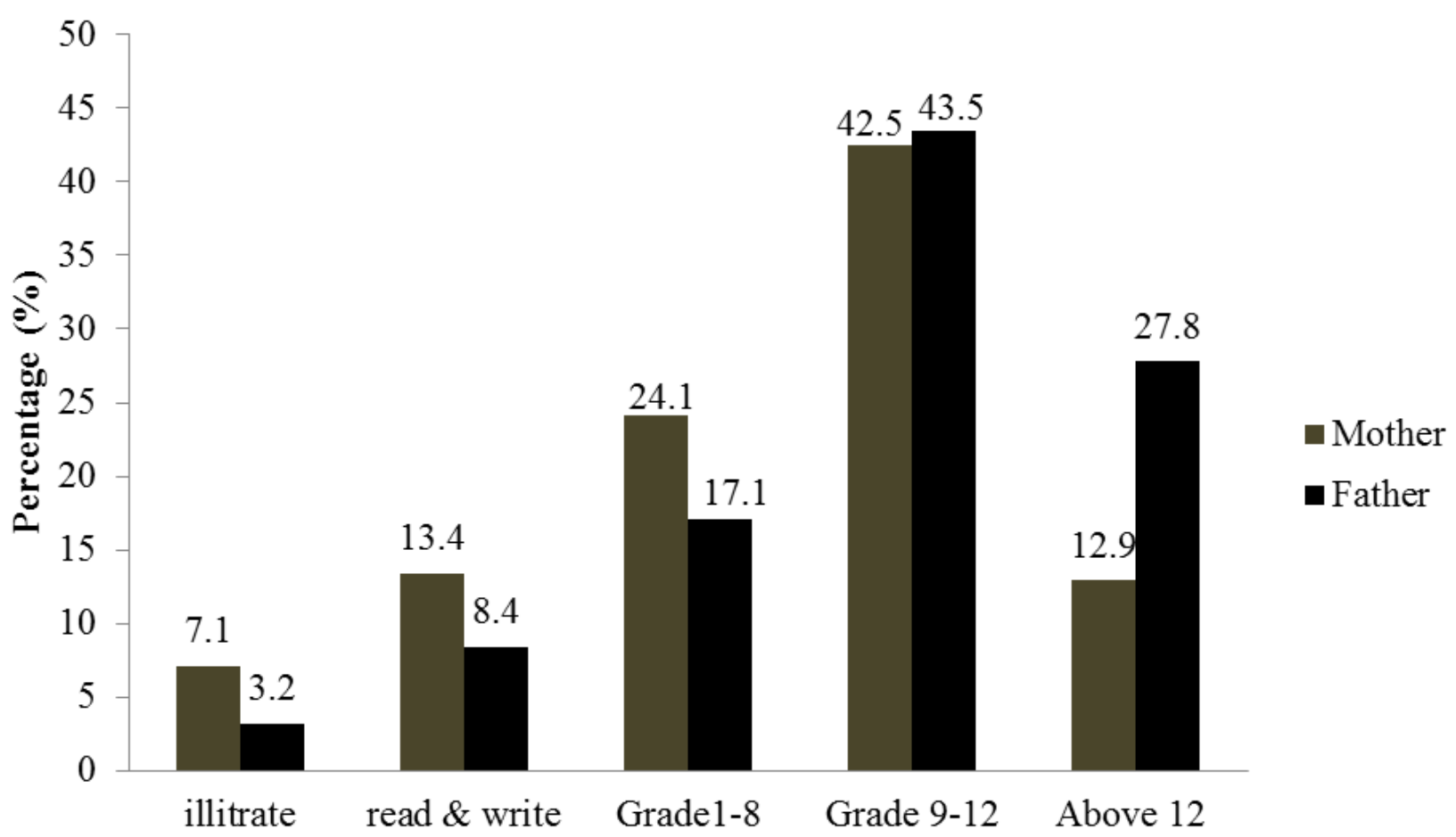

Fig. 2. The distribution of children by their parents' occupational status in Goba town, South eastern Ethiopia, May, 2012.

Concerning water source $323(81.4 \%)$ of the study subjects had access to tap water. Other drinking water sources were public stand pipe (bono) which accounts about 49 (12.3\%) and from river $25(6.3 \%)$.

The most commonly consumed staple foods were assessed and most $310(78.3 \%)$ of the study subjects reported that they were commonly provided bread with tea for breakfast, followed by Injera (thin bread made up of Teff) with Shiro wet which accounted for $65(16.4 \%)$ of the study subjects. Regarding the commonly consumed lunch and dinner food items, nearly all $369(92.9 \%)$ of the respondents reported that they ate Injera with Shiro wet. Concerning snack food items, most of them $236(68.6 \%)$ responded bread with tea as their staple foods for snack followed by Injera with shiro wet reported by 66(19.2\%) of the participants. They were also asked whether they have ever eaten fish to which most of them replied as never 256(64.5\%) and the remaining $140(35.3 \%)$ responded that they had eaten fish.

The goiter prevalence among the study subjects measured by palpation was $50.6 \%(95 \% \mathrm{CI}=(46.6,60.5)$ with $33.5 \%$ $\{95 \% \mathrm{CI}=(28.9,38.4)\}$ for Grade 1 goiter and $17.1 \%\{95 \%$ $\mathrm{CI}=(13.6,21.3)\}$ for grade 2 goiter. The prevalence of goiter was higher in females $112(53.6 \%)\{95 \% \mathrm{CI}=(30.9,39.1)\}$ than in males $89(47.3 \%)\{95 \% \mathrm{CI}=(16.3,23.1)\}$

During the survey, the availability of iodized salt in the households was determined. Children were asked to bring one tea spoon food salt and the salt was tested based on the standardized procedure. A high proportion of school children $169(42.6 \%)$ brought inadequately iodized salt $(<15 \mathrm{ppm})$. One hundred ten $(27.7 \%)$ of them brought salt which had no iodine $(0 \mathrm{ppm})$ and only $118(29.7 \%)$ of them brought salt which contained sufficient iodine ( $>15 \mathrm{ppm})$.
Table 1. Socio-demographic profile of elementary school children in Goba town, South East, Ethiopia May, 2012.

\begin{tabular}{lll}
\hline Variables & Frequency & Percentage \\
\hline Sex & 188 & \\
Male & 209 & 47.4 \\
Female & & 52.6 \\
Age & 82 & \\
6-8 & 315 & 20.7 \\
$9-12$ & & 79.3 \\
Religion & 83 & \\
Muslim & 279 & 20.9 \\
Orthodox & 35 & 70.3 \\
Protestant & & 8.8 \\
Ethnicity & 134 & \\
Amhara & 14 & 33.8 \\
Gurage & 230 & 3.5 \\
Oromo & 19 & 57.9 \\
Others & & 4.8 \\
Family income & 28 & \\
High & 272 & 7.1 \\
Medium & 97 & 68.5 \\
Low & & 24.4 \\
Number of rooms in house & 45 & \\
1 room & 123 & 11.3 \\
2 rooms & 133 & 31.0 \\
3 rooms & 96 & 33.5 \\
$>4$ rooms & & 24.3 \\
Number of people in home & 176 & \\
2-4 & 199 & 44.3 \\
5-7 & 22 & 50.1 \\
$8-11$ & & 5.5 \\
\hline & &
\end{tabular}

Others* Tigria, Wolaita, Somali

Binary and multiple logistic regression model was performed to identify the factors associated with goiter. Variables which were identified to have significant association 
at $\mathrm{p}$-value less than 0.05 with goiter in the bivariate regression model were entered into multiple logistic regression model. In multivariate logistic regression model, the age of school children $(\mathrm{AOR}=1.86 ; 95 \% \mathrm{CI}=1.10,3.15)$ and the number of individual living in the home $(\mathrm{AOR}=3.79 ; 95 \% \mathrm{CI}=1.37$, 10.48) had association with goiter (see Table 2).
The goiter prevalence was found higher in females than males though statistically insignificant in the studied school age children.

Other variables like sex, place of birth, educational status of father and mother and water source were found to have no significant association with goiter in the bivariate analysis.

Table 2. Associated factors with goiter among school children aged 6-12 years in Goba town, South East, Ethiopia, 2012.

\begin{tabular}{|c|c|c|c|c|}
\hline \multirow{2}{*}{ Variables } & \multicolumn{2}{|c|}{ Goiter status of children } & \multirow{2}{*}{$\begin{array}{l}\text { Crude OR } \\
(95 \% \text { CI })\end{array}$} & \multirow{2}{*}{$\begin{array}{l}\text { Adjusted OR } \\
(95 \% \text { CI })\end{array}$} \\
\hline & Yes & No & & \\
\hline \multicolumn{5}{|l|}{ Sex } \\
\hline Male & $89(47.3 \%)$ & $99(52.7 \%)$ & 1.0 & 1.0 \\
\hline Female & $112(53.6 \%)$ & $97(46.4)$ & $1.284(0.865,1.906)$ & $1.432(0.940,2.184)$ \\
\hline \multicolumn{5}{|l|}{ Age (year) } \\
\hline $6-8$ & $32(39 \%)$ & $50(61 \%)$ & 1.0 & 1.0 \\
\hline $9-12$ & $169(53.7 \%)$ & $146(46.3 \%)$ & $1.809(1.102,2.970)$ & $1.862(1.102,3.148)^{*}$ \\
\hline Out of Goba & $56(47.1 \%)$ & $63(52.9 \%)$ & 1.0 & 1.0 \\
\hline In Goba & $145(52.2 \%)$ & $133(47.8 \%)$ & $1.227(0.798,1.886)$ & $1.476(0.920,2.370)$ \\
\hline \multicolumn{5}{|l|}{ Water source } \\
\hline Pipe water & $166(51.4 \%)$ & $157(48.6 \%)$ & 1.0 & 1.0 \\
\hline Bono & $23(46.9 \%)$ & $26(53.1 \%)$ & $0.837(0.458,1.528)$ & $0.974(0.515,1.845)$ \\
\hline River & $12(48 \%)$ & $13(52 \%)$ & $0.873(0.387,1.971)$ & $0.835(0.353,1.978)$ \\
\hline \multicolumn{5}{|c|}{ Family income } \\
\hline Medium & $140(51.5 \%)$ & $132(48.5 \%)$ & $0.589(0.262,1.323)$ & $0.524(0.221,1.243)$ \\
\hline Low & $43(44.3 \%)$ & $54(55.7 \%)$ & $0.442(0.185,1.057)$ & $0.484(0.193,1.218)$ \\
\hline \multicolumn{5}{|c|}{ No people in home } \\
\hline $2-4$ & $70(39.8 \%)$ & $106(60.2 \%)$ & 1.0 & 1.0 \\
\hline $5-7$ & $115(57.8 \%)$ & $84(42.2 \%)$ & $2.073(1.372,3.132)$ & $2.231(1.453,3.424) * *$ \\
\hline $8-11$ & $16(72.7 \%)$ & $6(27.3 \%)$ & $4.038(1.507,10.820)$ & $3.793(1.372,10.484)^{* *}$ \\
\hline \multicolumn{5}{|l|}{ Salt test } \\
\hline$>15 \mathrm{ppm}$ & $60(50.8 \%)$ & $58(49.2 \%)$ & 1.0 & 1.0 \\
\hline$<15 \mathrm{ppm}$ & $87(51.5 \%)$ & $82(48.5 \%)$ & $1.026(0.641,1.642)$ & $1.056(0.642,1.737)$ \\
\hline $0 \mathrm{ppm}$ & $54(49.1 \%)$ & $56(50.9 \%)$ & $0.932(0.554,1.562)$ & $1.014(0.587,1.751)$ \\
\hline \multicolumn{5}{|c|}{ Fish ever consumption } \\
\hline Yes & $79(56.4 \%)$ & $61(43.6 \%)$ & 1.0 & 1.0 \\
\hline No & $121(47.3 \%)$ & $135(52.7 \%)$ & $0.692(0.457,1.048)$ & $0.705(0.453,1.096)$ \\
\hline
\end{tabular}

* Statistical significance at $\mathrm{p}<0.05$

** Statistical significance at $\mathrm{p}<0.01$

\section{Discussion}

The total prevalence of goiter in this study is $50.6 \%$ which is greater compared to other studies in Ethiopia [8, 9, 10]. However, this finding is almost equivalent to that of the study done nationwide in Ethiopia which was 50.3\% [5]. The total goiter prevalence in this study is greater than the study done in different parts of the world (11.4\% in India, $40 \%$ in Nepal, Tanzania $22 \%$ and Ecuador $30 \%$ ) $[11,12]$. This difference may be due to Ethiopia being a mountainous country and poor soil conservation over a long period contributed to the leaching away of the iodine-rich soil layer and the exposing of the iodine-poor layer beneath. This process is usually more severe in highland areas like our study area. Also, the relatively small sample size could contribute to the difference of goiter prevalence.

WHO recommends that a total goiter rate of $5 \%$ or more in primary school children of 6 to 12 years of age be used to signal the presence of a public health problem [4].

The prevalence in this study was higher in females $(46.4 \%)$ than in males $(30.9 \%)$. This is consistent with similar studies done in Ethiopia [8, 9]. The prevalence of goiter in males and females were $10.7 \%$ and $11.9 \%$ respectively in India and $7.4 \%$ and $15.7 \%$ respectively in Rajasthan $[13,14]$.however, there were no statistically significant gender differences on goiter prevalence in the studies both in Ethiopia and outside Ethiopia[8,9,13,14]. The higher goiter rate in girls than in boys is expected because of the physiological differences that increase the demand for extra iodine and the loss of iodine from the female body.

This study revealed that goiter prevalence increases with age (39 \% for $6-8$ years age group and $53.7 \%$ for $9-12$ years age group). This result is consistent with another study in India where the prevalence of goiter among 8, 9, and 10 year old children, were $10.8 \%, 11.0 \%$, and $12.0 \%$ respectively [13]. The same result was found in Tafilah governorate that the prevalence of goiter higher among 10 year old children than 8 year old children [15].

Salt iodization is by far the most important populationbased intervention for IDD control and has been shown to be efficacious in alleviating IDD assuming iodine 
concentrations in the salt are at appropriate levels at the time of consumption [16]. Consumption of iodized salt is the simplest and least expensive modality of IDD management. However, this research revealed that only 118 (29.7\%) of the study participants' families were using sufficiently iodized salt $(>15 \mathrm{ppm})$. This research result is almost comparable with the result of EDHS 2011 for urban households with 23\% using iodized salt [7]. Another study in Ethiopia showed that only $4.2 \%$ of the households had iodized salt [5].

Among the socio demographic variables the 9-12 age group and the number of household members were found to be highly associated with goiter $(\mathrm{AOR}=1.86 ; 95 \% \mathrm{CI}=1.10$, $3.15)$ and $(\mathrm{AOR}=3.79 ; 95 \% \mathrm{CI}=1.37,10.48)$ respectively. This result is is similar with other studies in Sodo town, Southern Ethiopia and southern part of Jordan [10, 17, 18].

\section{Limitations}

It is possible that variables such as genetic variations which were not measured in this study can be potential confounders. We were unable to measure urinary iodine level of the study subjects which would reveal recent iodine intake status. In addition, very young children may give false response for some questions in the interview.

\section{Conclusion and Recommendations}

The total prevalence of goiter among school children is very high. Therefore Goba town can be classified as endemic area for goiter. The relative proportion of goiter was higher among female students and the proportion increased with age. Grade 1 goitre was more prevalent than Grade 2 goiter. There was significant association of the goiter status of children with age and family size.The majority salt test result showed that food salt is not sufficiently iodized. Based on the result of this research work, the following recommendations are forwarded.

Continuous concerted efforts should be made to create awareness about the importance of iodized salt consumption, and periodic assessment of iodized salt regarding iodine content and the availability in the market through a regular monitoring system. Further studies on larger population sizes may be needed to confirm the status of IDD in Bale zone including the investigation of different causes of goiter other than iodine deficiency and the difference of academic performance between students with and without goiter. A household survey for goitre and consumption of iodized salt is needed to detect the presence of familial goitre and utilization of iodized salt.

\section{List of Abbreviations}

$\begin{array}{ll}\text { CBDC } & \text { Centers for Disease Control and Prevention } \\ \text { BSc } & \text { Bachelor of science } \\ \text { EDHS } & \text { Ethiopian Demographic Health Survey } \\ \text { ICCIDD } & \begin{array}{l}\text { International Council for Control of Iodine } \\ \text { Deficiency Disorders }\end{array}\end{array}$

$\begin{array}{ll}\text { IDD } & \text { Iodine Deficiency Disorders } \\ \text { IQ } & \text { Intelligence Quotient } \\ \text { MOH } & \text { Ministry of Health } \\ \text { SPSS } & \text { Statistical Package for Social Sciences } \\ \text { SNNP } & \text { Southern nation, nationalities and people } \\ \text { Tg } & \text { Thyroglobulin } \\ \text { TGP/R } & \text { Total Goiter Prevalence/Rate } \\ \text { TSH } & \text { Thyroid stimulating hormone } \\ \text { UIE } & \text { Urinary iodine excretion } \\ \text { UN } & \text { United Nations } \\ \text { UNICEF } & \text { United Nations Children's Fund } \\ \text { WHO } & \text { World Health Organization }\end{array}$

\section{Authors Contribution}

Habtamu Demelash designed the study, developed the questionnaire, supervised the data collection, analyzed the data and wrote the paper. Ketema Gashaw contributed to the development of the overall study concept, design of the study, the questionnaire, drafting of the paper and analysis of the data. Addisu Melese contributed to the interpretation of the findings as well as the drafting and writing of the manuscript. All authors read and approved the final manuscript.

\section{Acknowledgments}

We gratefully acknowledge the financial grants from Madawalabu University.

We are also grateful to thank UNICEF (nutrition unit) in Ethiopia for their provision of field iodine salt test kits. Moreover, we would also like to extend our thanks to all staffs of Medicine and Health Science College of Madawalabu University for their valuable comments and suggestions.

We would like to thank Goba town Education Office, and all staff members of Sof umer, Sanete, Arada Mincha and Finchan Bamo Elementary School for their cooperation and assistance during data collection.

Finally, we would like to forward our gratitude to the study participants and data collectors for their great contribution for the completion of this study.

\section{References}

[1] Mahshid L JBM. The Prevention and Control of Iodine Deficiency Disorders - Nutrition policy discussion paper Netherlands, 1988 Contract No.: 3.

[2] Souvik S. Prevalence of iodine deficiency disorders among schoolchildren in three blocks of Bardhhaman district and Bardhhaman municipal area of west Bengal, India, 2005.

[3] Iodine Deficiency Disorders and Intellectual Performance of School Children Aged 8 - 10 Years Old A Case Study in Malang District, East Java. 2005.

[4] WHO. Indictors for assessing iodine deficiency disorders and their control through salt iodization. Geneva1994 Contract No.: $\mathrm{WHO} / \mathrm{NUT} / 94.6)$. 
[5] Cherinet A YB, Girma A, Zewditu G, and Tesema E. Prevalence of goiter in children 6 to 12 years of age in Ethiopia. 2005 2-4.

[6] Minali S and Biswajit M. Iodine deficiency in school going children of Pondicherry. Indian j pediatre. 2007; 4(8):731-4.

[7] CSA. Ethiopian Demographic and Health survey, Addis Ababa, Ethiopia 2011(2):201-8.

[8] Cherinet A KU. Determinants of iodine deficiency in school children in different regions of ethiopia. 2000:35-41.

[9] Negalign Berhanu KWM, Mesele Bezabih. Endemic goiter in School Children in Southwestern Ethiopia. Ethiop J Health Dev2003; 18(3):177-9.

[10] Eskinder W. The effect of Iodine Deficiency Disorder on Academic performance of school children in Sodo town, Southern Ethiopia. 2011.

[11] Madhu B RM, Lakshminarayana J. Assessment of Iodine Deficiency Disorders in School Age Children in Jodhpur dictrict of Rajasthan, India, 2010.

[12] WHO. Assessment of iodine deficiency disorders and monitoring their elimination. 2007.
[13] Akhil Bandhu B IC, Dilip Kumar D, Srabani B, Saswati N, Jayasri M,. Iodine Deficiency Disorders among School Children of Malda, West Bengal, India. J HEALTH POPUL NUTR2002; 20(2):2-4.

[14] Madhu B SR, Marwal J, Lakshminarayana,. Assessment of Iodine Deficiency Disorders in School Age Children in Jodhpur dictrict of Rajasthan. J Hum Ecol 2010; 32(2):80-5.

[15] Latifeh A MI, Hamed T, Mohamed N, Ahmed A,. prevalence of iodine deficiency among goiterous school children in Tafilah governorate JRMS 2005;12(2):42-

[16] Emel G OE, Günay C, Semra A, Șima G, Serdar C, Prevalence and Risk Factors of Iodine Deficiency among Schoolchildren in Turkey. Journal of Tropical Pediatrics1999; 49(3).

[17] Latifeh A S, mousa I,. Prevalence of iodine deficiency among goiterous school children, Jordan. 2005.

[18] Khairya M. National Study On The Prevalence of Iodine Deficiency Disorders Among School Children Aged 8-12 years old in Bahrai. 2000. 